\title{
MicroRNA-1 downregulation induced by carvedilol protects cardiomyocytes against apoptosis by targeting heat shock protein 60
}

\author{
YINGYING HU ${ }^{1,2}$, XI CHEN $^{1,2}$, XINA LI $^{1}$, ZHANGE LI $^{2}$, HONGTAO DIAO $^{2}$, LU LIU ${ }^{1}$, JIA ZHANG ${ }^{1}$, \\ JIN JU ${ }^{2}$, LIN WEN ${ }^{2}$, XIN LIU ${ }^{2}$, ZHENWEI PAN ${ }^{2}$, CHAOQIAN XU $^{3}$, XIN HAI $^{1}$ and YONG ZHANG ${ }^{2,4}$ \\ ${ }^{1}$ Department of Pharmacy, The First Affiliated Hospital of Harbin Medical University, Harbin, Heilongjiang 150001; \\ ${ }^{2}$ Department of Pharmacology, The State-Province Key Laboratories of Biomedicine-Pharmaceutics of China,
}

Key Laboratory of Cardiovascular Research, Ministry of Education, Harbin Medical University, Harbin, Heilongjiang 150081;

${ }^{3}$ Center of Chronic Diseases and Drug Research, Mudanjiang Medical University, Mudanjiang, Heilongjiang 157011;

${ }^{4}$ Institute of Metabolic Disease, Heilongjiang Academy of Medical Science, Harbin, Heilongjiang 150081, P.R. China

Received September 4, 2018; Accepted March 6, 2019

DOI: $10.3892 / \mathrm{mmr} .2019 .10034$

\begin{abstract}
Myocardial infarction (MI) is the most common event in cardiovascular disease. Carvedilol, a $\beta$-blocker with multiple pleiotropic actions, is widely used for the treatment cardiovascular diseases. However, the underlying mechanisms of carvedilol on alleviating MI are not fully understood. The aim of the present study was to investigate whether the beneficial effects of carvedilol were associated with regulation of microRNA-1 (miR-1). It was demonstrated that carvedilol ameliorated impaired cardiac function and decreased infarct size in a rat model of MI induced by coronary artery occlusion. Similarly, carvedilol reversed the $\mathrm{H}_{2} \mathrm{O}_{2}$-induced decrease in cardiomyocyte viability in a dose-dependent manner. The in vivo and in vitro models demonstrated the downregulation of miR-1 following treatment with carvedilol. Overexpression of miR-1, a known pro-apoptotic miRNA, decreased cell viability and induced cell apoptosis. Transfection of miR-1 abolished the beneficial effects of carvedilol. The expression
\end{abstract}

Correspondence to: Professor Xin Hai, Department of Pharmacy, The First Affiliated Hospital of Harbin Medical University, 23 Youzheng Street, Harbin, Heilongjiang 150001, P.R. China

E-mail: hai_xin@163.com

Professor Yong Zhang, Department of Pharmacology, The State-Province Key Laboratories of Biomedicine-Pharmaceutics of China, Key Laboratory of Cardiovascular Research, Ministry of Education, Harbin Medical University, 157 Baojian Road, Harbin, Heilongjiang 150081, P.R. China

E-mail: hmuzhangyong@hotmail.com

Abbreviations: miR-1, microRNA-1; MI, myocardial infarction; HSP60, heat shock protein 60; Bcl-2, B-cell lymphoma 2; Bax, Bcl-2-associated $\mathrm{X}$ protein

Key words: myocardial infarction, apoptosis, cardiomyocyte, carvedilol, microRNA-1, heat shock protein 60 of heat shock protein 60 (HSP60), a direct target of miR-1, was identified to be decreased in $\mathrm{MI}$ and $\mathrm{H}_{2} \mathrm{O}_{2}$-induced apoptosis, which was associated with a decrease in Bcl-2 and an increase in Bax; expression was restored following treatment with carvedilol. It was concluded that carvedilol partially exhibited its beneficial effects by downregulating miR-1 and increasing HSP60 expression. miR-1 has become a member of the group of carvedilol-responsive miRNAs. Future studies are required to fully elucidate the potential overlapping or compensatory effects of known carvedilol-responsive miRNAs and their underlying mechanisms of action in the pathophysiology of cardiovascular diseases.

\section{Introduction}

Myocardial infarction (MI) due to coronary occlusion is the most common event in cardiovascular disease $(1,2)$. Following acute MI, cardiomyocyte apoptosis occurs; apoptosis may be an indispensable pathway in cardiomyocyte death during acute ischemia $(3,4)$. The inhibition of cardiomyocyte apoptosis following MI may be an effective method to ameliorate left ventricular remodeling and promote cardiac function.

Carvedilol, a nonselective $\beta$-adrenergic receptor ( $\beta$-AR) antagonist, exhibits multiple pleiotropic properties, including $\alpha$-adrenergic receptor ( $\alpha$-AR) blocking and antioxidative activities $(5,6)$. Several studies using a variety of in vitro and in vivo models have provided evidence for the cardioprotective role of carvedilol; Nakamura et al (7) demonstrated that the administration of carvedilol improved cardiac function by decreasing oxidative stress levels. In addition, carvedilol decreased cardiomyocytic apoptosis by suppressing the expression of inflammation-associated genes and apoptosis-associated proteins through the phosphoinositide 3-kinase- and mitogen activated protein kinase kinase-associated signaling pathways (8). However, the exact underlying mechanisms of carvedilol are yet to be fully determined.

MicroRNAs (miRNAs) are a class of endogenous small non-coding RNAs measuring 22 nucleotides that negatively 
regulate gene expression at the post-transcriptional level via the 3'-untranslated region (3'-UTR) of target mRNAs. Previously, an emerging role of miRNAs in the development of cardiovascular diseases has been explored $(9,10)$. Among the known miRNAs, cardiac-enriched and muscle-specific miR-1 has been demonstrated to be a key regulator of cardiac development and disease (11-14). With the exception of regulating cardiac development, miR-1 has been proposed to be involved in regulating cardiomyocyte apoptosis. Notably, miR-1 levels were significantly increased in response to oxidative stress-induced apoptosis (15).

The aim of the present study was to investigate whether carvedilol protects cardiomyocytes from apoptosis in an MI rat model. Whether $\mathrm{H}_{2} \mathrm{O}_{2}$-induced cardiomyocyte apoptosis may be associated with the effects of miR-1 expression under the pathological conditions of cardiac disease was also determined.

\section{Materials and methods}

Animal models of myocardial infarction and drug administration. Prior to the initiation of the experimental procedures, 30 healthy male Wistar rats (250-300 g) were obtained from The Animal Center of the 2nd Affiliated Hospital of Harbin Medical University (Harbin, China). Rats were housed under temperature- $\left(23 \pm 1^{\circ} \mathrm{C}\right)$ and humidity- $(55 \pm 5 \%)$ controlled conditions, with food and water ad libitum for 1 week. The rats used in the present study were randomly divided into the sham, myocardial infarction and carvedilol (Car) groups. As described previously, left anterior descending coronary artery ligation was performed to induce myocardial infarction (16). The rats in the Car group were pretreated with an oral dose of $10 \mathrm{mg} / \mathrm{kg}$ carvedilol daily for 14 days prior to surgery. The rats in the sham group underwent open chest procedures without coronary artery occlusion. The rats were anesthetized with pentobarbital sodium $(40 \mathrm{mg} / \mathrm{kg}$ ) by intraperitoneal injection prior to surgery and transthoracic echocardiography. The physical methods of cervical dislocation or decapitation were performed for euthanasia of the adult or neonatal rats, respectively. Consistent with the American Veterinary Medical Association Guidelines for the Euthanasia of Animals (2013 Edition) (17), death was confirmed prior to disposal of the rats. A combination of criteria, including lack of pulse, breathing, corneal reflex or response to firm toe pinch, inability to hear respiratory sounds and heartbeat by use of a stethoscope, graying of the mucous membranes and rigor mortis were used to verify animal death.

Echocardiography and infarct area assessment. Transthoracic echocardiography was performed $24 \mathrm{~h}$ after coronary artery ligation to measure the left ventricular internal dimension in M-mode. Following echocardiography, ventricular tissues were collected and stored at $-20^{\circ} \mathrm{C}$ for $20 \mathrm{~min}$. The samples were then sliced into $2-\mathrm{mm}$ thick sections and incubated in $1 \%$ 2,3,5-triphenyltetrazolium chloride (Beijing Solarbio Science \& Technology, Beijing, China) at $37^{\circ} \mathrm{C}$ in $0.2 \mathrm{M}$ Tris buffer (pH 7.4) for $30 \mathrm{~min}$. Then, the sections were placed on clean paper for imaging. Infarct size was assessed by examining the extracted hearts; the ratio of average scar size to the average left ventricular size was calculated. All quantitative evaluations were performed using ImagePro Plus v6.0 software (Media Cybernetics, Inc., Rockville, MD, USA).

Cell culture and transfection. The hearts of 1- to 3-day-old neonatal Wistar rats were isolated and ground in serum-free Dulbecco's modified Eagle's medium (HyClone; GE Healthcare Life Sciences, Logan, UT, USA), and then incubated with $0.25 \%$ trypsin solution until the tissues were almost completely digested. After $2 \mathrm{~h}$, non-adherent cardiomyocytes were collected and re-plated in 6-well plates supplemented with $10 \%$ fetal bovine serum (HyClone; GE Healthcare Life Sciences) and $100 \mu \mathrm{g} / \mathrm{ml}$ penicillin/streptomycin at $5 \% \mathrm{CO}_{2}$ and $37^{\circ} \mathrm{C}$ for $48 \mathrm{~h}$. Prior to all experiments, cardiomyocytes were serum-starved overnight. Subsequently, cardiomyocytes were treated with $100 \mu \mathrm{M} \mathrm{H}_{2} \mathrm{O}_{2}$ or $10 \mu \mathrm{M}$ carvedilol (Bikai Pharmaceutical Co., Ltd., Haikou, China) for 24 h, or transfected with $50 \mathrm{nM}$ miR-1 (5'-UGGAAUGUAAAGAAGUAU GUAU-3'), miR-1 inhibitor (5'-AUACAUACUUCUUUA CAUUCCA-3') or negative control (5'-UUUGUACUACACA AAAGUACUG-3'), which were synthesized by Guangzhou RiboBio Co., Ltd. (Guangzhou, China). miRs were transfected using X-treme GENE siRNA transfection reagent (Roche Diagnostics, Basel, Switzerland) according to the manufacturer's protocol. After $12 \mathrm{~h}$ of transfection, cardiomyocytes were treated with $\mathrm{H}_{2} \mathrm{O}_{2}$ or carvedilol.

Cell viability assay. An MTT assay was used to determine cell viability. Cardiomyocytes at a density of $2 \times 10^{4}$ cells/well were plated into 96-well plates and transfected, and/or treated with carvedilol or $\mathrm{H}_{2} \mathrm{O}_{2}$. Following incubation with $15 \mu \mathrm{l}$ MTT for $4 \mathrm{~h}$ at $37^{\circ} \mathrm{C}$, the culture medium was carefully removed. Then, $150 \mu \mathrm{l}$ dimethyl sulfoxide was added to each well. The plates were agitated to dissolve the formazan crystals. Finally, the absorbance was measured at $490 \mathrm{~nm}$ using an Infinite M200 Pro microplate spectrophotometer (Tecan Group, Ltd., Männedorf, Switzerland).

Identification of target gene. The potential target genes of miR-1 were predicted using TargetScanHuman (version 7.2; http://www.targetscan.org/vert_72/).

Protein extraction and western blot analysis. Total protein samples were extracted from the left ventricular peri-infarct region of rats or cardiomyocytes as described previously (18). Briefly, homogenized specimens or cells were lysed in $300 \mu \mathrm{l}$ of radioimmunoprecipitation assay buffer (cat. no. R0020; Beijing Solarbio Science \& Technology) containing 1\% protease inhibitor. Then centrifuging at $12,000 \mathrm{x}$ g for $25 \mathrm{~min}$ at $4^{\circ} \mathrm{C}$. The protein concentration in the supernatant was examined using a bicinchoninic acid protein assay (Beyotime Institute of Biotechnology, Haimen, China). A total of $100 \mu \mathrm{g}$ protein was loaded in each lane. Proteins were separated by $10 \%$ SDS-PAGE. Following transfer of the proteins to Pure Nitrocellulose Blotting membranes (Pall Life Sciences, Port Washington, NY, USA), the blots were probed with primary antibodies against HSP60 (1:1,000, cat. no. 12165S; Cell Signaling Technology, Inc., Danvers, MA, USA), B-cell lymphoma 2 (Bcl-2; 1:1,000; cat. no. 26593-1-AP; ProteinTech Group, Inc., Chicago, IL, USA), Bcl-2-associated $\mathrm{X}$ protein (Bax; 1:1,000; cat. no. 50599-2-Ig; ProteinTech 
Group, Inc., Chicago, IL, USA) and GAPDH (1:1,000; cat. no. KC-5G4; Chengdu Kang Cheng Electronic Co., Ltd., Chengdu, China), which was the internal control. Membranes were blocked using 5\% skim milk (cat. no. 232100; San Jose, CA, USA) at $4^{\circ} \mathrm{C}$ overnight. Membranes were subsequently incubated with IRDye $800 \mathrm{CW}$-labeled goat anti-rabbit IgG (1:10,000; cat. no. 926-32211; LI-COR Biosciences) and IRDye $800 \mathrm{CW}$-labeled goat anti-mouse $\operatorname{IgG}(1: 10,000$; cat. no. 926-32210; LI-COR Biosciences) for $1 \mathrm{~h}$ at room temperature. Protein expression was analyzed and quantified using Odyssey Infrared Imaging System (version 3.0; LI-COR Biosciences).

Reverse transcription quantitative polymerase chain reaction $(R T-q P C R)$. Total RNA samples were extracted from heart tissues or cardiomyocytes using TRIzol ${ }^{\circledR}$ (Thermo Fisher Scientific, Inc., Waltham, MA, USA) according to the manufacturer's protocol. The extracted RNA was reverse transcribed into cDNA using a High-Capacity cDNA RT kit (cat. no. 4368814; Thermo Fisher Scientific, Inc.) according to the manufacturer's protocol. An RT primer containing a highly stable stem-loop structure was used to elongate the target miRNA. The plates were incubated for $15 \mathrm{~min}$ at $16^{\circ} \mathrm{C}$, for $1 \mathrm{~h}$ at $37^{\circ} \mathrm{C}$ and for $5 \mathrm{~min}$ at $85^{\circ} \mathrm{C}$. The expression levels of miR-1 and HSP60 mRNA were determined using a SYBR Green PCR master mix kit (cat. no. 4309155; Thermo Fisher Scientific, Inc.) on an ABI 7500 fast Real Time PCR system (Applied Biosystems; Thermo Fisher Scientific, Inc.). U6 and GAPDH were employed as the internal controls for miR-1 and HSP60, respectively. The cDNA samples were amplified in 96 -well plates for $10 \mathrm{~min}$ at $95^{\circ} \mathrm{C}$, followed by 40 cycles of $15 \mathrm{sec}$ at $95^{\circ} \mathrm{C}, 30 \mathrm{sec}$ at $60^{\circ} \mathrm{C}$ and $30 \mathrm{sec}$ at $72^{\circ} \mathrm{C}$. The relative expression of the miRNA and mRNA were determined using the $\mathrm{Cq}\left(2^{-\Delta \Delta \mathrm{Cq}}\right)$ quantification method (19). The sequences of the primers used were the following: miR-1 RT primer, 5'GTCGTATCCAGTGCGTGTCGTGGAGTCGGCAATTGC ACTGGATACGACATACACAC-3'; miR-1 forward, 5'-GGC TGGAATGTAAAGAAGTG-3'; miR-1 reverse, 5'-TATCCA GTGCGTGTCGTG-3'; U6 forward, 5'-GCTTCGGCAGCA CATATACTAAAAT-3'; U6 reverse, 5'-CGCTTCACGAAT TTGCGTGTCAT-3'. HSP60 forward, 5'- GGCTATCGCTAC TGGT-3'; HSP60 reverse, 5'- GCAAGTCGCTCGTTCA-3'; GAPDH forward, 5'-AAGAAGGTGGTGAAGCAGGC-3'; GAPDH reverse, 5'-TCCACCACCCAGTTGCTGTA-3'.

Terminal deoxynucleotidyl-transferase-mediated dUTP nick end labeling (TUNEL) assay. To evaluate the level of cell apoptosis, a TUNEL assay was performed using an In Situ Cell Death Detection kit (Roche Diagnostics GmbH, Mannheim, Germany) according to the manufacturer's protocols. Cardiomyocytes grown on coverslips were washed with PBS, and fixed in $4 \%$ paraformaldehyde solution for $1 \mathrm{~h}$ at $4^{\circ} \mathrm{C}$. The cells were permeabilized in a solution containing $0.1 \%$ Triton $\mathrm{X}-100$ for $2 \mathrm{~min}$, followed by incubation in freshly prepared TUNEL reaction mixture for $1 \mathrm{~h}$ at $37^{\circ} \mathrm{C}$ in the dark. Subsequently, the TUNEL-stained coverslips were washed with PBS and then counterstained with DAPI (1:20 dilution; Beyotime Institute of Biotechnology) for $15 \mathrm{~min}$ at room temperature. Nuclei double-labelled with TUNEL and DAPI were considered to be TUNEL-positive as detected in $\geq 5$ fields of view under a laser confocal microscope (Olympus Corporation, Tokyo, Japan; magnification, x200).

Statistical analysis. The data are presented as mean \pm standard error of the mean. Statistical comparisons among multiple groups were performed using a one-way analysis of variance, followed by a Bonferroni's multiple comparison post-hoc test. Statistical values were analyzed using GraphPad Prism 5.0 (GraphPad Software, Inc., La Jolla, CA, USA). P<0.05 was considered to indicate a statistically significant difference.

\section{Results}

Carvedilol ameliorates impaired cardiac function and decreases infarct size. In our previous study, it was identified that carvedilol ameliorated impaired cardiac function within infarcted rats (20). Consistent with a previous study, echocardiography was performed $24 \mathrm{~h}$ post-MI induction, prior to 2 weeks of treatment with carvedilol. Analysis demonstrated that MI hearts were significantly dilated, as evidenced by an increase in the left ventricular systolic internal dimension, indicating impaired cardiac function; no significant changes in left ventricular diastolic internal dimension were observed (Fig. 1A and B). Carvedilol decreased the left ventricular systolic internal dimension. The infarct size of MI rats was significantly increased, whereas the size of infarcted area was decreased by $20 \%$ following the administration of carvedilol (Fig. 1C and D).

Carvedilol increases cardiomyocyte viability and inhibits miR-1 expression. In the present study, $100 \mu \mathrm{M} \mathrm{H}_{2} \mathrm{O}_{2}$ and $10 \mu \mathrm{M}$ carvedilol were the treatments selected for subsequent experiments. It was identified that $\mathrm{H}_{2} \mathrm{O}_{2}$ significantly decreased cardiomyocyte viability, whereas treatment with carvedilol reversed decreases in cell viability induced by $\mathrm{H}_{2} \mathrm{O}_{2}$ (Fig. 2A). To investigate whether miR-1 expression was altered following carvedilol treatment, RT-qPCR was performed to detect the miR-1 expression levels in myocardial tissue and cardiomyocytes. The expression levels of miR-1 were significantly increased by $\sim 45 \%$ in MI myocardial tissues, but were significantly downregulated in the MI + Car group compared with the MI group (Fig. 2B). Similarly, in cultured cardiomyocytes, upregulation of miR-1 expression induced by $\mathrm{H}_{2} \mathrm{O}_{2}$ was significantly reversed following treatment with carvedilol (Fig. 2C).

Overexpression of miR-1 induces cardiomyocyte apoptosis. According to the aforementioned results above, we hypothesized that the anti-apoptotic effect of carvedilol may be mediated by downregulating miR-1 expression. Therefore, the effects of miR-1 on cardiomyocytes were verified. Fig. 3A demonstrated that miR-1 was successfully transfected into cardiomyocytes. Overexpression of miR-1 significantly decreased cell viability, while no change was observed following transfection of the negative control (NC) (Fig. 3B). Notably, overexpression of miR-1 substantially induced cardiomyocyte apoptosis, as determined by TUNEL staining (Fig. 3C). In addition, no significant effects on cardiomyocyte apoptosis in the NC group were observed (Fig. 3C).

Downregulation of miR-1 is involved in the anti-apoptotic action of carvedilol. As indicated in Fig. 4A, treatment with 

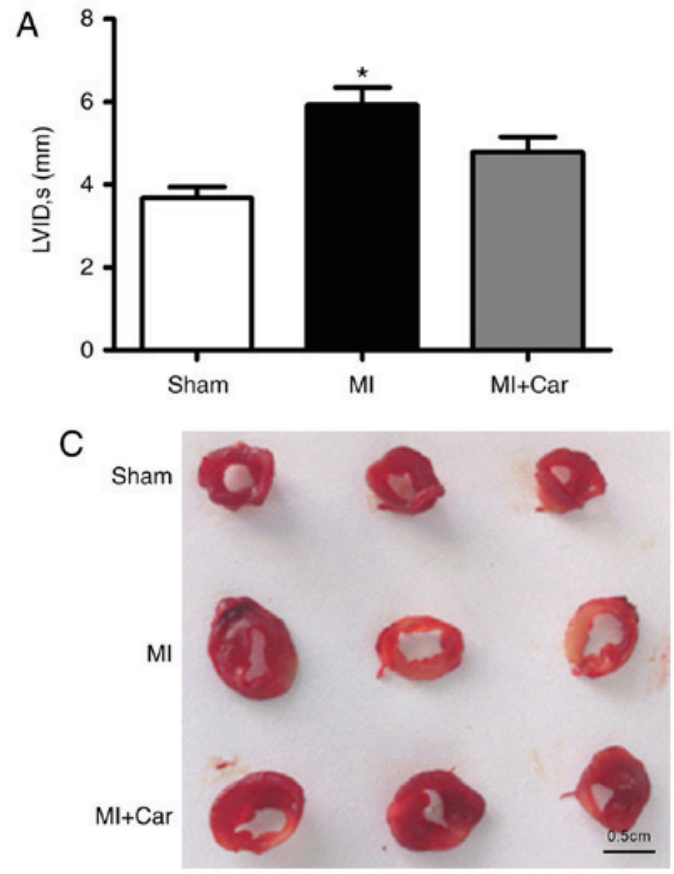
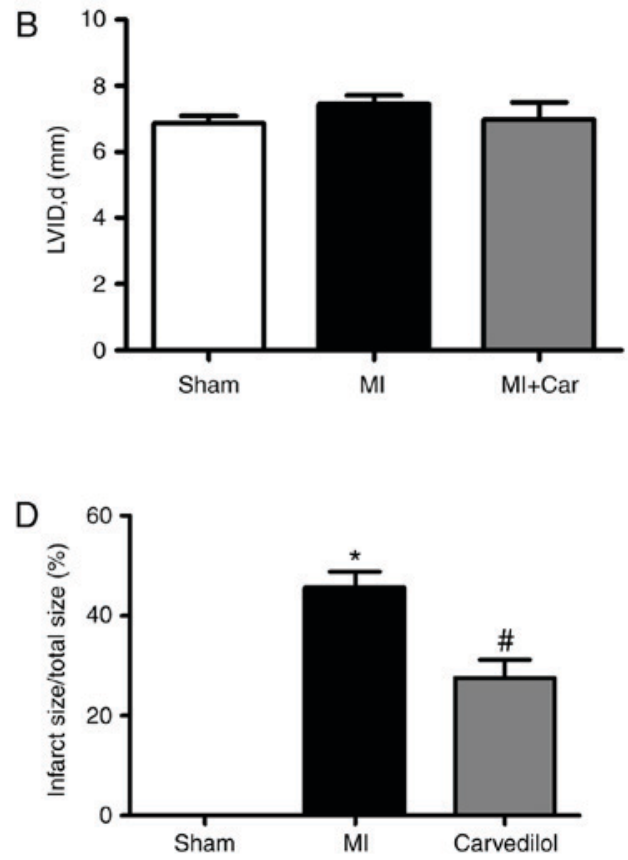

Figure 1. Effects of carvedilol on cardiac function and infarct size in a rat model of MI. MI was established by coronary artery ligation for $24 \mathrm{~h}$. (A) LVID, s, and (B) LVID, d, values in each model group. (C) Examples of infarcted area of the left ventricular walls, as indicated by 2,3,5-triphenyltetrazolium chloride staining. Normal areas were stained brick-red, and the infarcted areas remained unstained (white). Scale bar $=0.5 \mathrm{~cm}$. (D) Statistical analysis of infarct size from 3 rats. "P<0.05 vs. Sham; "P $<0.05$ vs. MI. MI, myocardial infarction; LVID, s, left ventricular systolic internal dimension; LVID, d, left ventricular diastolic internal dimension.
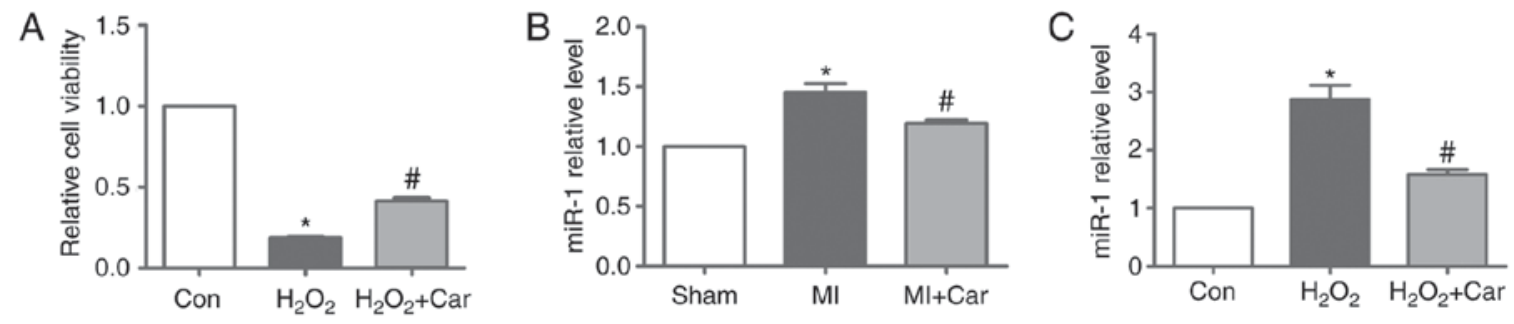

Figure 2. Effects of carvedilol on cardiomyocyte viability and miR-1 expression. (A) Effects of $10 \mu \mathrm{M}$ carvedilol on cardiomyocyte viability in the presence of $100 \mu \mathrm{M} \mathrm{H}_{2} \mathrm{O}_{2}$ for $24 \mathrm{~h}$, as determined by an MTT assay. (B) miR-1 expression in myocardial tissue as determined by RT-qPCR, which was normalized to U6. (C) miR-1 expression in cardiomyocytes as determined by RT-qPCR, which was normalized to U6. All data are presented as relative levels. "P $<0.05$ vs. Sham or Con; " $\mathrm{P}<0.05$ vs. MI or $\mathrm{H}_{2} \mathrm{O}_{2}$. miR, microRNA; RT-qPCR, reverse transcription quantitative polymerase chain reaction; car, carvedilol; con, control; MI, myocardial infarction.

carvedilol reversed the decrease in cell viability induced by $\mathrm{H}_{2} \mathrm{O}_{2}$, and the effect was almost eliminated under the condition of miR-1 transfection. Additionally, in Fig. 4B, TUNEL staining indicated that the $\mathrm{H}_{2} \mathrm{O}_{2}$-induced apoptosis of cardiomyocytes was attenuated by carvedilol. As expected, this was reversed by miR-1, indicating that the observed anti-apoptotic effect of carvedilol was mediated by down-regulation of miR-1.

Targeting HSP60 by miR-1 is a mechanism underlying the cytoprotection of carvedilol. To elucidate the molecular mechanisms by which miR-1 executes its function, HSP60 was bioinformatically predicted to be the conservative target of miR-1 using the bioinformatics tool TargetScan. The potential binding sites identified in the 3'UTR of the human, rat and mouse HSP60 gene for miR-1 are demonstrated in Fig. 5A. It was identified that the mRNA and protein expression levels of HSP60 were significantly decreased under the conditions of MI, while their levels were increased following pretreatment with carvedilol (Fig. 5B and C). $\mathrm{H}_{2} \mathrm{O}_{2}$ treatment markedly inhibited the expression of HSP60 at the mRNA and protein levels in vitro. These effects were notably inhibited by carvedilol, whereas cooperation with miR-1 eliminated the effects of carvedilol (Fig. 5D and E). Carvedilol alone also decreased the expression of miR-1 (Fig. 6A), and increased the mRNA expression levels of HSP60 (Fig. 6B).

Upregulation of HSP60 by carvedilol is associated with an increase in Bcl-2 and a decrease in Bax. As demonstrated in Fig. 7A, it was observed that carvedilol abrogated the $\mathrm{H}_{2} \mathrm{O}_{2}$-induced downregulation of $\mathrm{Bcl}-2$ protein expression. Furthermore, transfection of miR-1 resulted in the inability of carvedilol to upregulate the expression of Bcl-2. Conversely, the expression of Bax, a pro-apoptotic factor, was decreased by carvedilol treatment in the presence of $\mathrm{H}_{2} \mathrm{O}_{2}$, whereas 

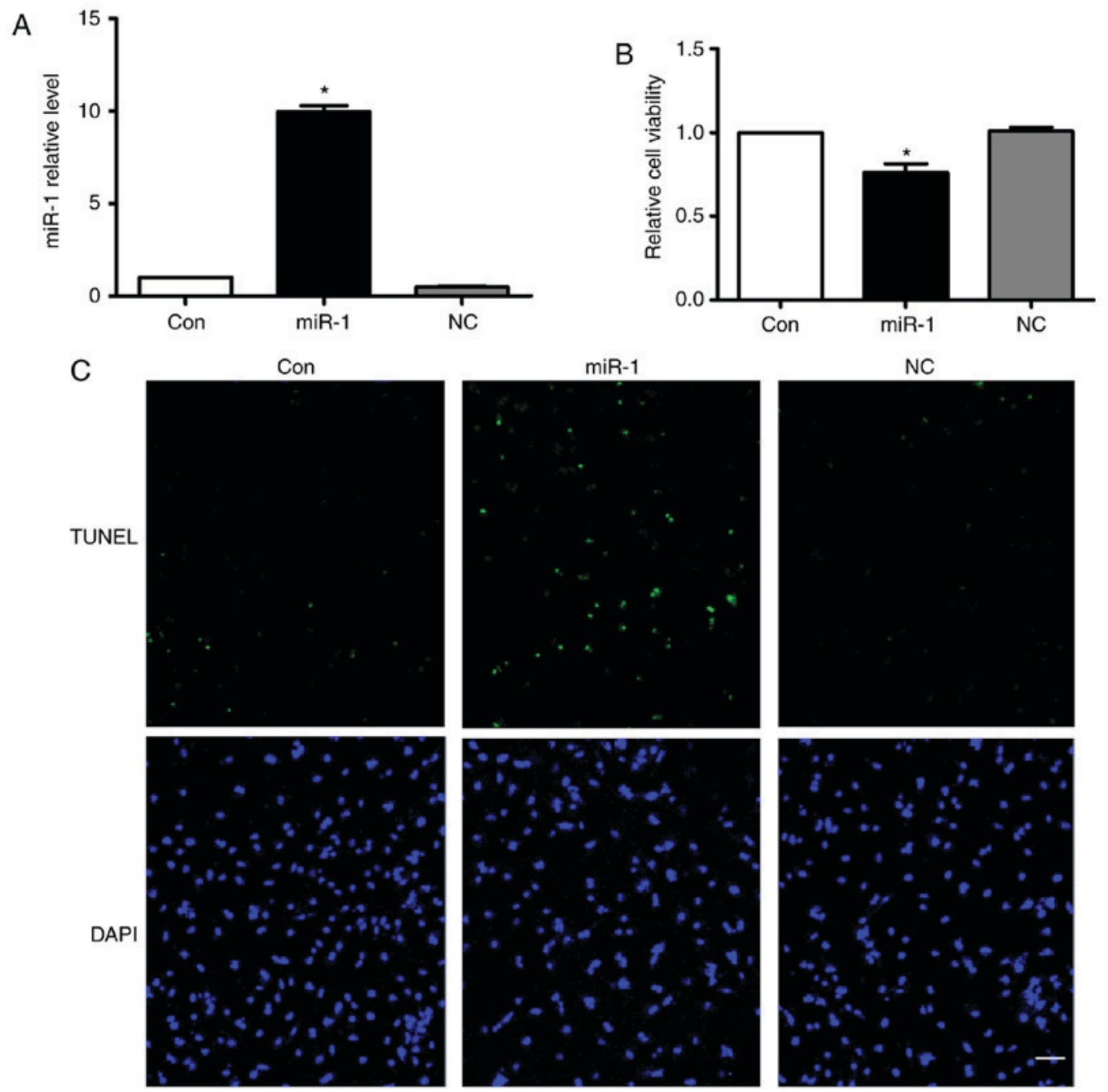

Figure 3. Overexpression of miR-1 induces the apoptosis of cardiomyocytes. (A) The miR-1 expression levels in cardiomyocytes at $24 \mathrm{~h}$ after transfection of miR-1 or $\mathrm{NC}$ as measured by reverse transcription quantitative polymerase chain reaction. ${ }^{*} \mathrm{P}<0.05 \mathrm{vs}$. Con. (B) Effect of miR-1 on cell viability as determined by an MTT assay. All data are presented as relative levels. "P<0.05 vs. Con. (C) TUNEL staining for apoptotic cells under different conditions. Scale bar=20 $\mu \mathrm{m}$. miR, microRNA; con, control; NC, negative control; TUNEL, terminal deoxynucleotidyl-transferase-mediated dUTP nick end labeling.

transfection of miR-1 reversed the effects of carvedilol (Fig. 7B).

\section{Discussion}

The mechanism underlying the interaction between miR-1 and carvedilol in MI may be associated with the decreased expression of miR-1, which occurred following treatment with carvedilol in the MI heart model of the present study, which implies a specific association between carvedilol and miR-1. Based on the results of the present study, a model of $\mathrm{H}_{2} \mathrm{O}_{2}$-induced cardiomyocyte apoptosis was established. It was identified that carvedilol protected cardiomyocytes against $\mathrm{H}_{2} \mathrm{O}_{2}$-induced apoptosis, which was accompanied with the downregulation of miR-1 expression; overexpression of miR-1 reversed the effects of carvedilol. Bioinformatic analysis revealed that HSP60 was a direct target of miR-1. Subsequent experiments suggested that the increased expression of HSP60 may be involved in the mechanism underlying the effects of carvedilol. Myocardial infarction is a progressive process, yet the underlying mechanism remains unknown. These data demonstrated that the downregulation of miR-1, at least in part, mediated the cytoprotective effects of carvedilol against cardiomyocyte apoptosis, which provides novel insight into the roles of miRNAs in the cardioprotective effects against ischemia exhibited by the $\beta$-blocker carvedilol.

It has been suggested that $\beta$-blockers exhibit highly effective roles in the treatment of cardiovascular diseases, including hypertension, coronary heart disease, angina, myocardial infarction and heart failure $(21,22)$. Carvedilol, a third-generation $\beta$-blocker, provided improved protection against vascular events compared with other $\beta$-blockers $(6,23,24)$. However, the underlying mechanism of this protection is not fully understood. Previous studies suggested that these protective effects observed in a number of cardiovascular diseases were due to the antioxidative properties of carvedilol (25-27). Carvedilol, a specific agonist of the $\beta 1$ - and $\beta 2$-ARs, selectively activates $\beta$-arrestin-mediated signaling $(28,29)$. Similarly, carvedilol has been demonstrated to activate EGFR-ERK signaling, which protects cardiomyocytes in a cell model system via $\beta$-arrestin 1 (28). 


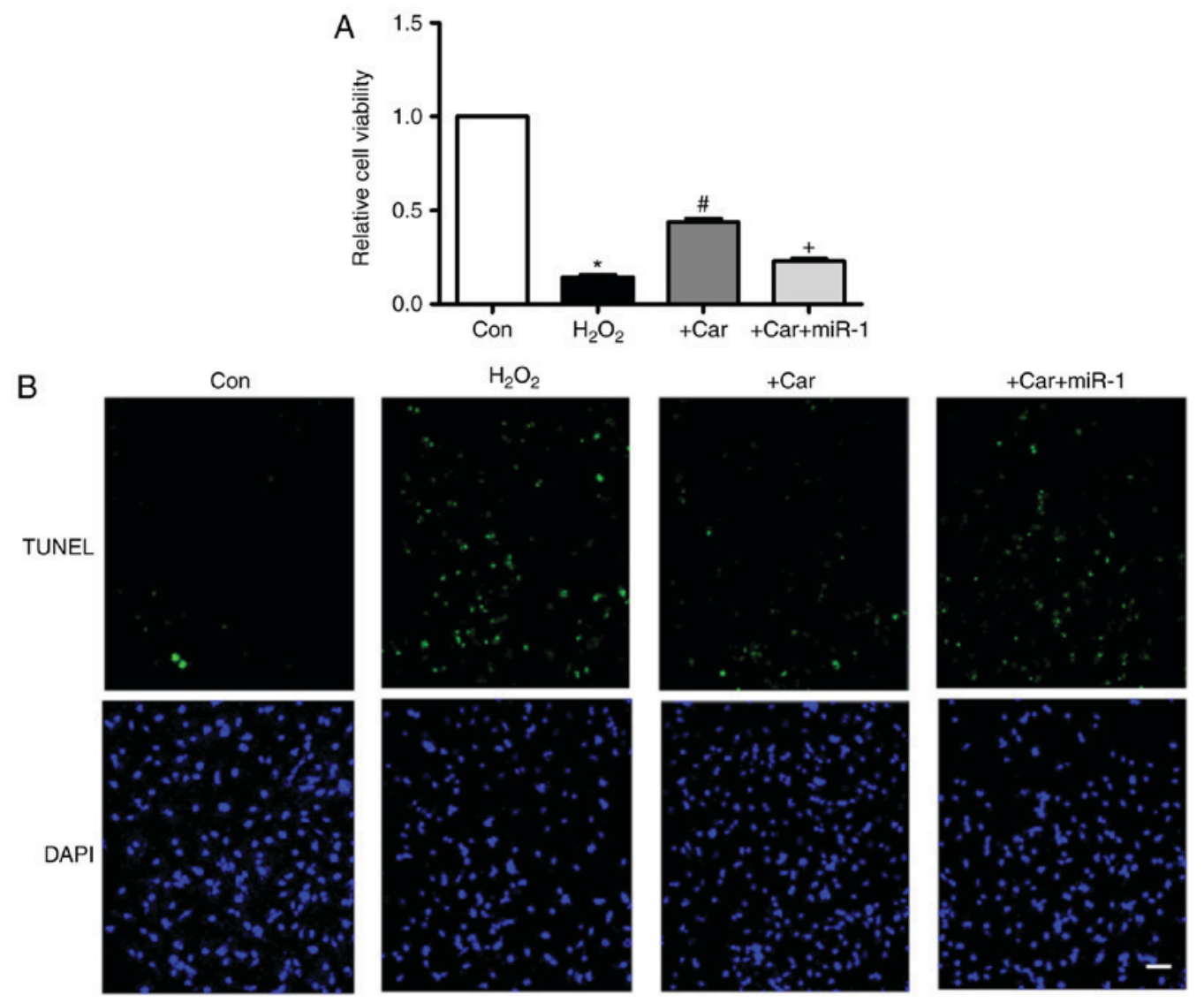

Figure 4. Transfection of miR-1 inhibits the beneficial effects of carvedilol. (A) Cardiomyocytes were pre-treated with vehicle or miR-1 for $12 \mathrm{~h}$, and then treated with $\mathrm{H}_{2} \mathrm{O}_{2}(100 \mu \mathrm{M})$ or carvedilol $(10 \mu \mathrm{M})$ for $24 \mathrm{~h}$. An MTT assay was conducted under different conditions to assess cell viability. All data are presented as relative levels. ${ }^{*} \mathrm{P}<0.05$ vs. Con; ${ }^{\prime} \mathrm{P}<0.05$ vs. $\mathrm{H}_{2} \mathrm{O}_{2} ;{ }^{+} \mathrm{P}<0.05$ vs. + Car. (B) TUNEL staining was used to detect apoptotic cardiomyocytes under different conditions. Scale bar indicates $20 \mu \mathrm{m}$. miR, microRNA; con, control; car, carvedilol; TUNEL, terminal deoxynucleotidyl-transferase-mediated dUTP nick end labeling.

Heat shock proteins, a group of molecular chaperones, are capable of preventing protein damage and proteolysis, which may be induced by heat, ischemia, hypothermia and hypoxia (30). HSP60, an important member of the heat shock protein family, is an anti-apoptotic protein expressed in mammalian hearts. HSP60 forms complexes with Bax, Bcl-2 homologous antagonist killer (Bak) and Bcl-xS, preventing Bax oligomerization and insertion into the mitochondrial membrane, thereby circumventing the mitochondrial apoptotic pathway $(12,30,31)$. HSP60 served a significant role in the recovery of mitochondrial function and the protection of cardiomyocytes. HSP60 protected against the apoptosis of cardiomyocytes by increasing the activities of complex III and IV in mitochondria, and decreasing the release and activity of mitochondrial cytochrome c and caspase-3, respectively, following ischemic stress $(32,33)$. In the present study, downregulated HSP60 was sufficient to induce apoptosis, which was associated with a decrease in $\mathrm{Bcl}-2$ and an increase in Bax expression, as previously demonstrated (31). However, carvedilol reversed the aforementioned effects to protect cardiomyocytes against apoptosis.

Several studies have demonstrated that miRNAs serve important roles in cardiovascular development and its associated diseases. For example, the cardiac-enriched, muscle-specific miR-1 has been identified for its critical role in myocardial infarction: Shan et al (34) indicated that
miR-1 and miR-206 participated in cardiomyocyte apoptosis in MI by suppressing insulin-like growth factor 1 . In addition, our previous study revealed that the upregulation of miR-1 delayed cardiac conduction and depolarized the cytoplasmic membrane via potassium voltage-gated channel subfamily $\mathrm{J}$ member 2 and gap junction protein alpha 1 gene downregulation, which encode the $\mathrm{K}^{+}$channel subunit Kir2.1 and gap junctional channel connexin 43 , respectively (35). Overexpression of miR-1 may also contribute to the increased susceptibility of the heart to atrioventricular block during the onset of myocardial ischemia (13). In the present study, it was identified that the $\beta$-blocker carvedilol protected against cardiomyocyte apoptosis by downregulating miR-1 in vivo and in vitro. This is consistent with the results from a previous study, which indicated the protective mechanism of $\beta$-AR blockers and tanshinone IIA in cardiomyocytes in ischemia.

Xu et al (20) first proposed that carvedilol exhibited cardioprotective effects against infarction and oxidative stress, which were associated with the increased expression levels of miR-133. These results from previous studies, and those from the present study, indicated that the combined actions of miR-1 and miR-133 associated with carvedilol produced beneficial cardioprotective effects. The two different mechanisms may function together to protect the heart from myocardial infarction, and may occur in various pathological conditions. 
A

\section{HSP60}

Human 235-241 of HSPD1 3' UTR

5' ...AUAAAAAACAUUUGUACAUUCCU...

hsa-miR-1

| | | | | ||

Rat 231-237 of HSPD1 3' UTR

$3^{\prime}$

UAUGUAUGAAGAAAUGUAAGGU

rno-miR-1

5' ...AAUAAAGACAUUUGUACAUUCCU...

$3^{\prime}$

I | I I I II

Mouse 231-237 of HSPD1 3' UTR

5' ...AAUAAAGACAUUUGUACAUUCCU...

mmu-miR-1a

3'

| | | | | |

B

C

HSP60 mRNA

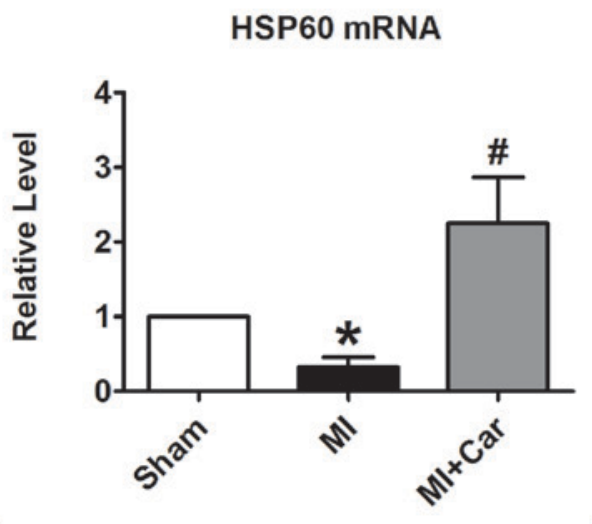

D

\section{E}
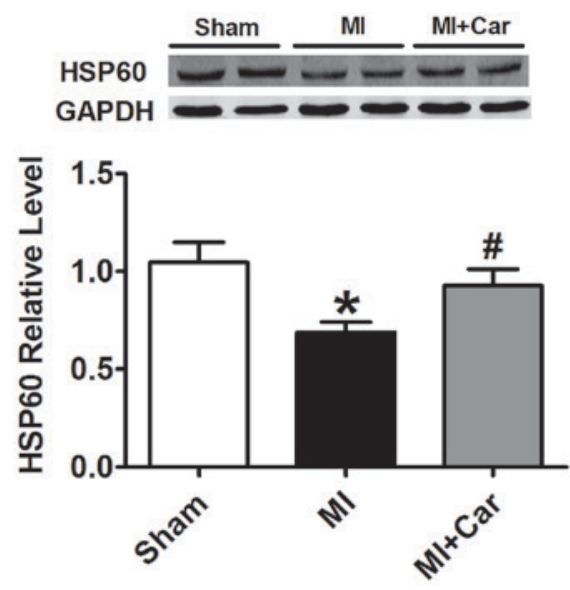

HSP60 MRNA
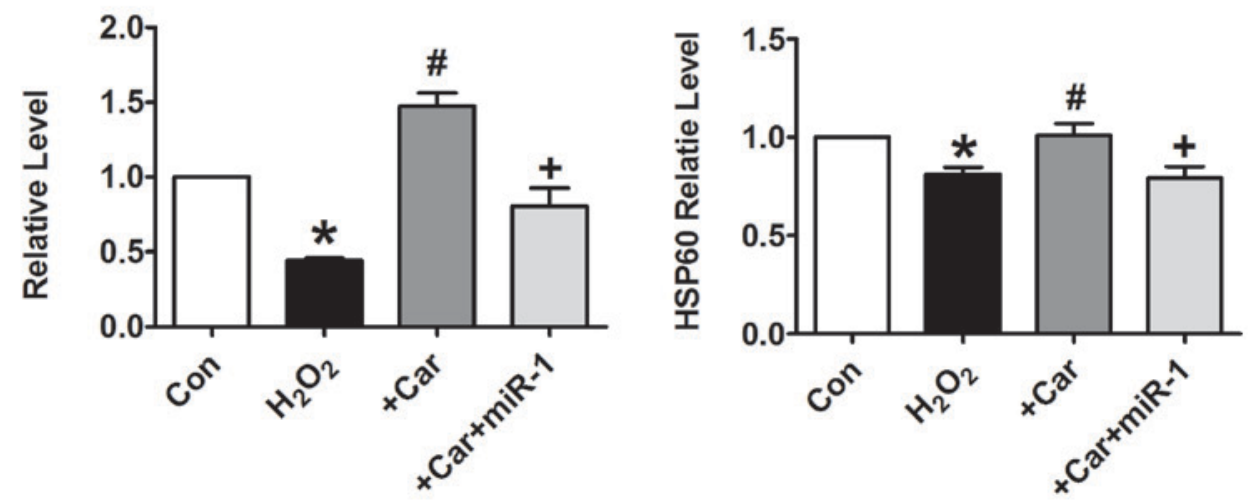

Figure 5. miR-1 suppresses the expression of HSP60 by binding to its 3'UTR. (A) Sequence alignment between miR-1 and the 3'UTR of human, rat and mouse HSP60. The matched base pairs are connected by a vertical line. (B) Effects of carvedilol on HSP60 mRNA levels in myocardial tissues, as measured by RT-qPCR. (C) Effects of carvedilol on HSP60 protein expression levels in myocardial tissues, as measured by western blot analysis. (D) Effects of carvedilol on HSP60 mRNA levels in cardiomyocytes, as measured by RT-qPCR. (E) Effects of carvedilol on HSP60 protein expression levels in cardiomyocytes, as measured by western blot analysis. Expression levels were normalized to that of GAPDH. All data are presented as relative levels. * $<0.05$ vs. Sham or Con; ${ }^{\#} \mathrm{P}<0.05$ vs. $\mathrm{MI}$ or $\mathrm{H}_{2} \mathrm{O}_{2} ;{ }^{+} \mathrm{P}<0.05$ vs. +Car. Car, carvedilol; Con, control; MI, myocardial infarction; HSPD1/HSP60, heat shock protein 60; miR, microRNA; 3'UTR, 3'untranslated region; RT-qPCR, reverse transcription quantitative polymerase chain reaction.

However, how carvedilol suppresses $\mathrm{H}_{2} \mathrm{O}_{2}$-induced increases in miR-1 and decreases in miR-133 expression remains unknown. Additional studies are required to fully understand the protective mechanisms of carvedilol under the conditions of ischemic injury.
In addition to miR-1 and miR-133, the association between carvedilol and associated miRNAs in the negative and positive regulation of cardiovascular diseases has been described in several studies (36-40). miR-125a-5p, miR-125b-5p, miR-150, miR-199a-3p and miR-214 were upregulated by carvedilol 

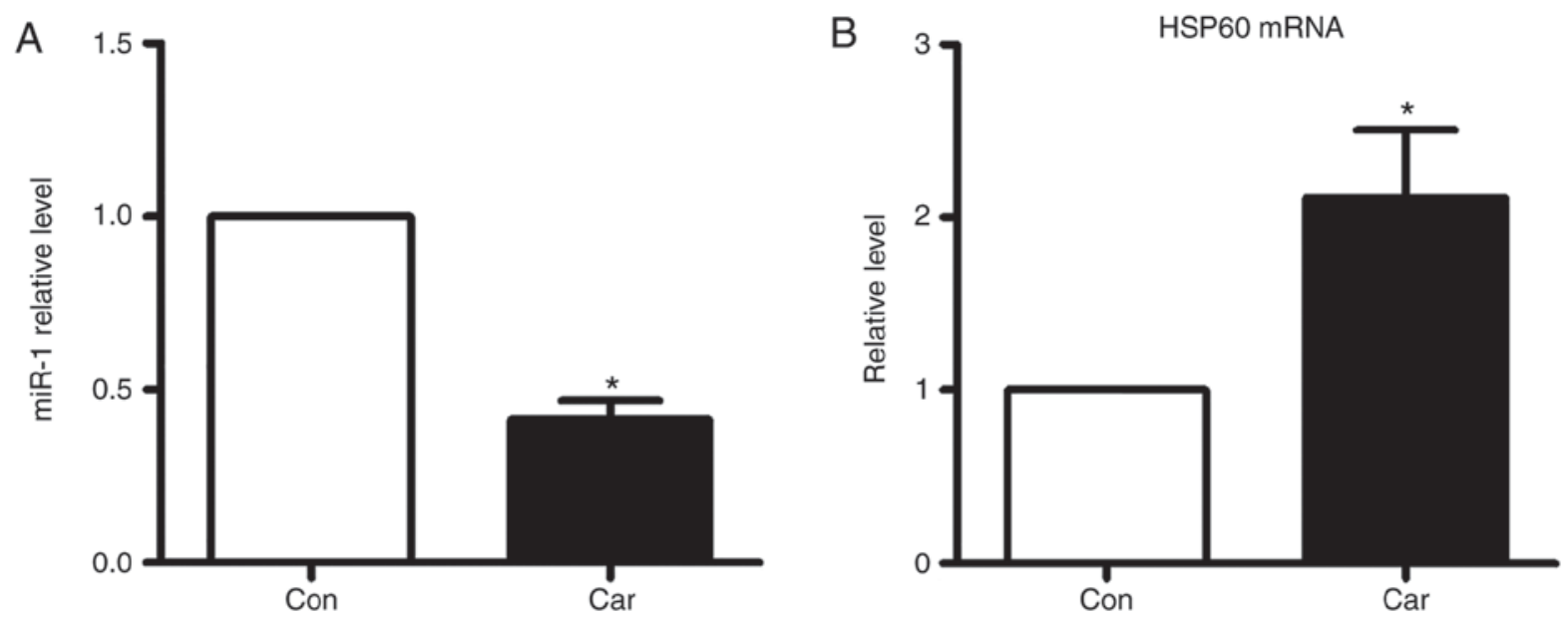

Figure 6. Effects of carvedilol alone on miR-1 and HSP60 expression. Reverse transcription quantitative polymerase chain reaction was applied to measure the mRNA expression levels of (A) miR-1 and (B) HSP60. All data are presented as relative levels. "P<0.05 vs Con. Car, carvedilol; Con, control; HSP60, heat shock protein $60 ;$ miR, microRNA.

A
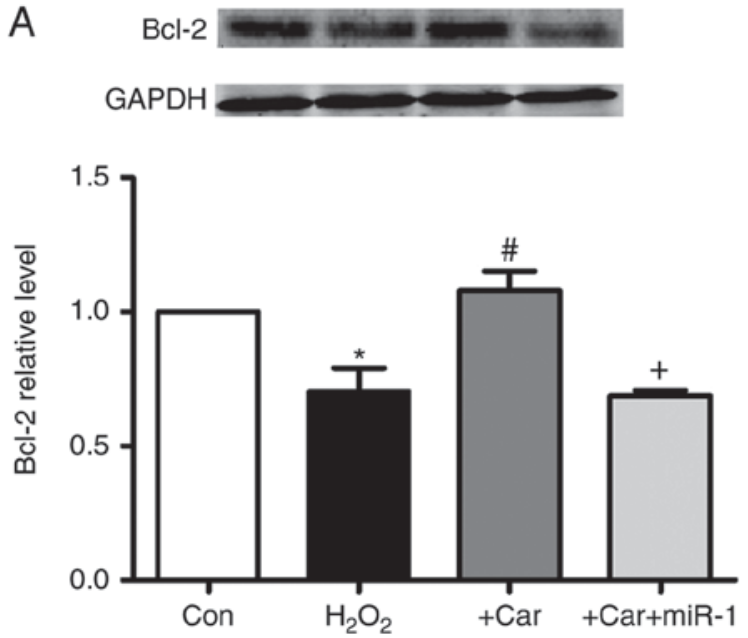

B
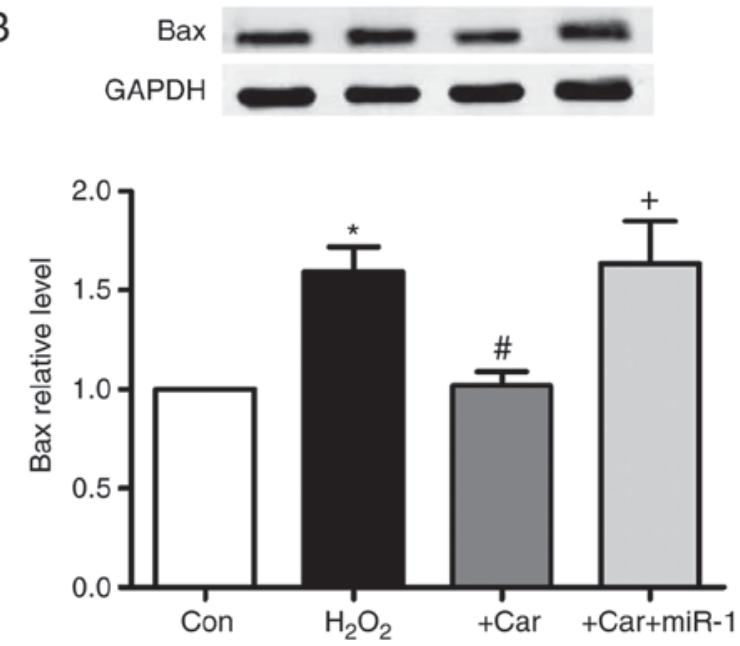

Figure 7. Upregulation of HSP60 by carvedilol is associated with increased Bcl-2 and decreased Bax expression. Effects of carvedilol on (A) Bcl-2 and (B) Bax protein expression levels in cardiomyocytes as measured by western blot analysis. Expression levels were normalized to GAPDH. All data are presented as relative levels. ${ }^{*} \mathrm{P}<0.05$ vs. Con; ${ }^{\#} \mathrm{P}<0.05$ vs. $\mathrm{H}_{2} \mathrm{O}_{2}$; ${ }^{+} \mathrm{P}<0.05$ vs. +Car. Bcl-2, B-cell lymphoma 2; Bax, Bcl-2-associated X protein; Car, carvedilol; Con, control; miR, microRNA.

stimulation; this effect was not observed in cells or mice lacking $\beta 1-A R, G$ protein-coupled receptor kinase $5 / 6$ or $\beta$-arrestin 1 (36). miR-125b-5p protected the heart against acute MI by inhibiting the death of cultured cardiomyocytes in response to injury, partially through the suppression of the pro-apoptotic genes Bcl-2 antagonist/killer 1 and Krüppel like factor 13 (37). Carvedilol-mediated upregulation of miR-466g or miR-532-5p was identified to be dependent on $\beta 2-A R s$, and upregulation of miR-674 by carvedilol was identified to be dependent on $\beta 1$-ARs (38). $\beta 2$-AR/ $\beta$-arrestin-responsive miR-532 conferred cardioprotection against MI though serving as a gatekeeper of cardiac vascularization by repressing a known endothelial-to-mesenchymal transition initiator, serine protease 23 (39). The upregulation of miR-29b, an additional cardioprotective miR, was demonstrated to contribute to the effects of carvedilol by attenuating post-MI fibrosis (40). Collectively, these studies support the hypothesis that the cardioprotective effects of carvedilol are associated with changes in the expression levels of carvedilol-responsive miRNAs.

It should be noted that the present study does not provide insight into the mechanism of action of carvedilol and miR-1. Previous studies have suggested that $\beta$-AR/ $\beta$-arrestin signaling is involved in miRNA maturation regulatory network activated by carvedilol, which may be associated with the protective effects of this therapeutic agent $(36,38)$. Therefore, there is an urgent requirement for additional investigations into the effects of gain or loss-of-function of the regulatory mechanism proposed in the present study in the pathophysiology of cardiovascular diseases.

In summary, the present study revealed that carvedilol protected cardiomyocytes from apoptosis by inhibiting miR-1 expression in cardiomyocytes; this cardioprotective effect was associated with increased HSP60 expression within cardiomyocytes in ischemia. Carvedilol-responsive miRNAs are increasingly recognized. Therefore, future studies are required 
to fully elucidate the potential overlapping/compensatory effects of known carvedilol-responsive miRNAs and their underlying mechanisms of action in the pathophysiology of cardiovascular diseases.

\section{Acknowledgements}

Not applicable.

\section{Funding}

The present study was supported in part by the Fund of Scientific Research Innovation of The First Affiliated Hospital of Harbin Medical University (grant no., 2016Y005), the National Natural Science Foundation of China (grant no., 81570399), and the Program for New Century Excellent Talents in Heilongjiang Provincial University (grant no., 1254-NCET-012).

\section{Availability of data and materials}

The datasets used and/or analyzed during the current study are available from the corresponding author on reasonable request.

\section{Authors' contribution}

$\mathrm{YZ}, \mathrm{XH}$ and $\mathrm{YH}$ conceived and designed the study. YH, XC, XL, ZL, HD, LL, JZ, JJ, LW, XL, ZP and CX performed data acquisition and analysis. YH wrote the manuscript. All authors reviewed and approved the manuscript.

\section{Ethics approval and consent to participate}

The present study was approved by the regulations of Experimental Animal Ethic Committee of Harbin Medical University, China (Animal Experimental Ethical Inspection Protocol No. 2010102), and conformed to the Guide for the Care and Use of Laboratory Animals published by the US National Institutes of Health (NIH Publication No. 85-23, revised 1996).

\section{Patient consent for publication}

Not applicable.

\section{Competing interests}

The authors declare that they have no competing interests.

\section{References}

1. Writing Group Members, Mozaffarian D, Benjamin EJ, Go AS Arnett DK, Blaha MJ, Cushman M, Das SR, de Ferranti S, Després JP, et al: Heart disease and stroke statistics-2016 update: A report from the American Heart Association. Circulation 133: e38-e360, 2016.

2. Sun T, Dong YH, Du W, Shi CY, Wang K, Tariq MA, Wang JX and Li PF: The role of microRNAs in myocardial infarction: From molecular mechanism to clinical application. Int $\mathrm{J}$ Mol Sci 18: pii: E745, 2017.

3. Liu Q, Zhang J, Xu Y, Huang Y and Wu C: Effect of carvedilol on cardiomyocyte apoptosis in a rat model of myocardial infarction: A role for toll-like receptor 4. Indian J Pharmacol 45: 458-463, 2013 .
4. Palojoki E, Saraste A, Eriksson A, Pulkki K, Kallajoki M, Voipio-Pulkki LM and Tikkanen I: Cardiomyocyte apoptosis and ventricular remodeling after myocardial infarction in rats. Am J Physiol Heart Circ Physiol 280: H2726-H2731, 2001.

5. Oliveira PJ, Marques MP, Batista de Carvalho LA and Moreno AJ: Effects of carvedilol on isolated heart mitochondria: Evidence for a protonophoretic mechanism. Biochem Biophys Res Commun 276: 82-87, 2000.

6. Wang R, Miura T, Harada N, Kametani R, Shibuya M, Fukagawa Y, Kawamura S, Ikeda Y, Hara M and Matsuzaki M: Pleiotropic effects of the beta-adrenoceptor blocker carvedilol on calcium regulation during oxidative stress-induced apoptosis in cardiomyocytes. J Pharmacol Exp Ther 318: 45-52, 2006.

7. Nakamura K, Kusano K, Nakamura Y, Kakishita M, Ohta K, Nagase S, Yamamoto M, Miyaji K, Saito H, Morita H, et al: Carvedilol decreases elevated oxidative stress in human failing myocardium. Circulation 105: 2867-2871, 2002.

8. Yeh CH, Chen TP, Wang YC, Lin YM and Fang SW: Carvedilol treatment after myocardial infarct decreases cardiomyocytic apoptosis in the peri-infarct zone during cardioplegia-induced cardiac arrest. Shock 39: 343-352, 2013.

9. De Rosa S, Curcio A and Indolfi C: Emerging role of microRNAs in cardiovascular diseases. Circ J 78: 567-575, 2014.

10. Latronico MV and Condorelli G: MicroRNAs and cardiac pathology. Nat Rev Cardiol 6: 419-429, 2009.

11. Pan Z, Sun X, Ren J, Li X, Gao X, Lu C, Zhang Y, Sun H, Wang Y, Wang $H$, et al: miR-1 exacerbates cardiac ischemia-reperfusion injury in mouse models. PLoS One 7: e50515, 2012.

12. Shan ZX, Lin QX, Deng CY, Zhu JN, Mai LP, Liu JL, Fu YH, Liu XY, Li YX, Zhang YY, et al: miR-1/miR-206 regulate Hsp60 expression contributing to glucose-mediated apoptosis in cardiomyocytes. FEBS Lett 584: 3592-3600, 2010.

13. Zhang Y, Sun L, Zhang Y, Liang H, Li X, Cai R, Wang L, Du W, Zhang R, Li J, et al: Overexpression of microRNA-1 causes atrioventricular block in rodents. Int J Biol Sci 9: 455-462, 2013.

14. Zhang Y, Zhang L, Chu W, Wang B, Zhang J, Zhao M, Li X, Li B, Lu Y, Yang B and Shan H: Tanshinone IIA inhibits miR-1 expression through p38 MAPK signal pathway in post-infarction rat cardiomyocytes. Cell Physiol Biochem 26: 991-998, 2010.

15. Tang Y, Zheng J, Sun Y, Wu Z, Liu Z and Huang G: MicroRNA-1 regulates cardiomyocyte apoptosis by targeting Bcl-2. Int Heart J 50: 377-387, 2009.

16. Lu Y, Zhang Y, Shan H, Pan Z, Li X, Li B, Xu C, Zhang B, Zhang F, Dong D, et al: MicroRNA-1 downregulation by propranolol in a rat model of myocardial infarction: A new mechanism for ischaemic cardioprotection. Cardiovasc Res 84: 434-441, 2009.

17. AVMA Quidelines for the Euthanasia of Animals: 2013 Edition. American Veterinary Medical Association, Schaumburg, IL, 2013. https://www.avma.org/KB/Policies/Documents/euthanasia.pdf. Accessed June 15, 2016.

18. Zhang Y, Li X, Zhang Q, Li J, Ju J, Du N, Liu X, Chen X, Cheng F, Yang L, et al: Berberine hydrochloride prevents postsurgery intestinal adhesion and inflammation in rats. J Pharmacol Exp Ther 349: 417-426, 2014.

19. Livak KJ and Schmittgen TD: Analysis of relative gene expression data using real-time quantitative PCR and the 2(-Delta Delta C(T)) method. Methods 25: 402-408, 2001.

20. Xu C, Hu Y, Hou L, Ju J, Li X, Du N, Guan X, Liu Z, Zhang T, Qin W, et al: $\beta$-Blocker carvedilol protects cardiomyocytes against oxidative stress-induced apoptosis by up-regulating miR-133 expression. J Mol Cell Cardiol 75: 111-121, 2014.

21. Kurdi M and Booz GW: Carvedilol protects the infarcted heart by upregulating miR-133: First evidence that disease state affects $\beta$-adrenergic arrestin-biased signaling? J Mol Cell Cardiol 76: 12-14, 2014

22. Poirier L and Tobe SW: Contemporary use of $\beta$-blockers: Clinical relevance of subclassification. Can J Cardiol 30 (5 Suppl): S9-S15, 2014.

23. Poole-Wilson PA, Swedberg K, Cleland JG, Di Lenarda A Hanrath P, Komajda M, Lubsen J, Lutiger B, Metra M, Remme WJ, et al: Comparison of carvedilol and metoprolol on clinical outcomes in patients with chronic heart failure in the carvedilol or metoprolol European trial (COMET): Randomised controlled trial. Lancet 362: 7-13, 2003.

24. Remme WJ, Torp-Pedersen C, Cleland JG, Poole-Wilson PA, Metra M, Komajda M, Swedberg K, Di Lenarda A, Spark P, Scherhag A, et al: Carvedilol protects better against vascular events than metoprolol in heart failure: Results from COMET. J Am Coll Cardiol 49: 963-971, 2007. 
25. Budni P, Pedrosa RC, Garlet TR, Dalmarco EM, Dalmarco JB, Lino MR, Simionato EL, Amara JA, Frode TS and Wilhelm Filho D: Carvedilol attenuates oxidative stress in chronic chagasic cardiomyopathy. Arq Bras Cardiol 98: 218-224, 2012 (In English).

26. Li YC, Ge LS, Yang PL, Tang JF, Lin JF, Chen P and Guan XQ: Carvedilol treatment ameliorates acute coxsackievirus B3-induced myocarditis associated with oxidative stress reduction. Eur J Pharmacol 640: 112-116, 2010.

27. Zhuang XF, Yin CQ, Wang HY and Sun NL: Distinctive effects of carvedilol in the non-infarct zone: Remodelling of the ligated rat heart linked to oxidative stress. J Int Med Res 37: 1354-1364, 2009.

28. Kim IM, Tilley DG, Chen J, Salazar NC, Whalen EJ, Violin JD and Rockman HA: Beta-blockers alprenolol and carvedilol stimulate beta-arrestin-mediated EGFR transactivation. Proc Natl Acad Sci USA 105: 14555-14560, 2008.

29. Wisler JW, DeWire SM, Whalen EJ, Violin JD, Drake MT, Ahn S, Shenoy SK and Lefkowitz RJ: A unique mechanism of beta-blocker action: Carvedilol stimulates beta-arrestin signaling. Proc Natl Acad Sci USA 104: 16657-16662, 2007.

30. Gupta S and Knowlton AA: HSP60, Bax, apoptosis and the heart. J Cell Mol Med 9: 51-58, 2005.

31. Kirchhoff SR, Gupta S and Knowlton AA: Cytosolic heat shock protein 60 , apoptosis, and myocardial injury. Circulation 105 2899-2904, 2002.

32. Hollander JM, Lin KM, Scott BT and Dillmann WH: Overexpression of PHGPx and HSP60/10 protects against ischemia/reoxygenation injury. Free Radic Biol Med 35: 742-751, 2003.

33. Lin KM, Lin B, Lian IY, Mestril R, Scheffler IE and Dillmann WH: Combined and individual mitochondrial HSP60 and HSP10 expression in cardiac myocytes protects mitochondrial function and prevents apoptotic cell deaths induced by simulated ischemia-reoxygenation. Circulation 103: 1787-1792, 2001.

34. Shan ZX, Lin QX, Fu YH, Deng CY, Zhou ZL, Zhu JN, Liu XY, Zhang YY, Li Y, Lin SG and Yu XY: Upregulated expression of $\mathrm{miR}-1 / \mathrm{miR}-206$ in a rat model of myocardial infarction. Biochem Biophys Res Commun 381: 597-601, 2009.
35. Yang B, Lin H, Xiao J, Lu Y, Luo X, Li B, Zhang Y, Xu C, Bai Y, Wang $\mathrm{H}$, et al: The muscle-specific microRNA miR-1 regulates cardiac arrhythmogenic potential by targeting GJA1 and KCNJ2. Nat Med 13: 486-491, 2007.

36. Kim IM, Wang Y, Park KM, Tang Y, Teoh JP, Vinson J, Traynham CJ, Pironti G, Mao L, Su H, et al: $\beta$-arrestin1-biased $\beta 1$-adrenergic receptor signaling regulates microRNA processing. Circ Res 114: 833-844, 2014.

37. Bayoumi AS, Park KM, Wang Y, Teoh JP, Aonuma T, Tang Y, Su H, Weintraub NL and Kim IM: A carvedilol-responsive microRNA, miR-125b-5p protects the heart from acute myocardial infarction by repressing pro-apoptotic bak1 and klf13 in cardiomyocytes. J Mol Cell Cardiol 114: 72-82, 2018.

38. Teoh JP, Bayoumi AS, Aonuma T, Xu Y, Johnson JA, Su H, Weintraub NL, Tang Y and Kim IM: $\beta$-arrestin-biased agonism of $\beta$-adrenergic receptor regulates Dicer-mediated microRNA maturation to promote cardioprotective signaling. J Mol Cell Cardiol 118: 225-236, 2018

39. Bayoumi AS, Teoh JP, Aonuma T, Yuan Z, Ruan X, Tang Y, Su H, Weintraub NL and Kim IM: MicroRNA-532 protects the heart in acute myocardial infarction, and represses prss 23 , a positive regulator of endothelial-to-mesenchymal transition. Cardiovase Res 113: 1603-1614, 2017.

40. Zhu JN, Chen R, Fu YH, Lin QX, Huang S, Guo LL, Zhang MZ, Deng CY, Zou X, Zhong SL, et al: Smad3 inactivation and MiR-29b upregulation mediate the effect of carvedilol on attenuating the acute myocardium infarction-induced myocardial fibrosis in rat. PLoS One 8: e75557, 2013.

(i) $(-)$ This work is licensed under a Creative Commons Attribution-NonCommercial-NoDerivatives 4.0 International (CC BY-NC-ND 4.0) License. 